\title{
AB/AC Stacking Boundaries in Bilayer Graphene
}

Junhao Lin ${ }^{1,2}$, Wu Zhou ${ }^{2}$, Wenjing Fang ${ }^{3}$, Jing Kong ${ }^{3}$, Andrew R. Lupini ${ }^{2}$, Juan-Carlos Idrobo ${ }^{2}$, Stephen J. Pennycook ${ }^{2,1}$, Sokrates T. Pantelides ${ }^{1,2}$

${ }^{1 .}$ Department of Physics and Astronomy, Vanderbilt University, Nashville, TN 37235, USA

2. Materials Science \& Technology Division, Oak Ridge National Laboratory, Oak Ridge, TN 37831, USA

3. Department of Electrical Engineering and Computer Science, Massachusetts Institute of Technology, Cambridge, MA 02139, USA

Boundaries, including grain boundaries and domain boundaries, are well known to modify the physical properties of materials. For example, it has been shown that certain types of grain boundaries in graphene can weaken the mechanical properties and degrade the electrical performance [1, 2]. Besides the well-studied grain boundaries, unique stacking boundaries can be present in bilayer graphene (BLG) at the interfaces of domains with the same crystal orientation but different stacking, i.e. $\mathrm{AB}$ and $\mathrm{AC}$ stacking. Recent studies have shown the coexistence of $\mathrm{AB}$ and $\mathrm{AC}$ stacking domains and the presence of domain boundaries [3]. However, little is known about the detailed atomic structure of these $\mathrm{AB} / \mathrm{AC}$ stacking boundaries formed in BLG. In particular, it is not known if the stacking boundaries are atomically sharp like grain boundaries.

A CVD-grown bilayer graphene sample is shown in Figure 1. Tilted dark-field transmission electron microscopy (DF-TEM) imaging at $80 \mathrm{kV}$ reveals the AB and AC stacking domains by their mirrorsymmetric intensity during tilting (Figure $1 \mathrm{a} \& 1 \mathrm{~b}$ ). Us ing the second order diffraction spot, the boundaries between the $\mathrm{AB}$ and $\mathrm{AC}$ stacking domains can be observed as dark lines in the DF-TEM image shown in Figure 1c. The decrease in DF-TEM im age intensity across the stacking boundaries suggests that the transition from $\mathrm{AB}$ to $\mathrm{AC}$ stacking takes place over a width of a few nanometers.

The atomic structure of stacking boundaries is further investigated using annular dark-field (ADF) imaging on an aberration-corrected scanning transmission electron microscope (STEM) operated at 60 $\mathrm{kV}$. The ADF image can be approximately interpreted as the convolution of the projected atomic positions in both graphene layers and the electron probe. While $\mathrm{AB}$ or $\mathrm{AC}$ stacking generates regular patterns with half of the carbon atoms overlapped, we observed irregular Moiré patterns at the stacking boundary regions. The precise pattern changes gradually across the stacking boundaries as shown in Figure 2. The observation demonstrates that the stacking boundaries are indeed continuous transitions from $\mathrm{AB}$ to $\mathrm{AC}$ stacking over a distance of several nanometers, which is consistent with the DF-TEM results.

Via density functional theory (DFT) and classical molecular dynamics calculations we further show that, unlike grain boundaries, the low-energy stacking boundaries are not s harp. Instead, they are nm-wide strain ripples that provide smooth transitions between the $\mathrm{AB}$ and $\mathrm{AC}$ stacking, in accord with the experimental observations. By optimizing the height, width and strain of the ripples using molecular dynamics, all the experimental STEM images can be reproduced by the simulated structural model (Figure 2a), confirming that the stacking boundaries exist as ripples. [4] 


\section{References:}

[1] O.V. Yazyev and S.G. Louie, Nature Materials, 9 (2010), 806.

[2] Y.J. Wei et al., Nature Materials, 11 (2012), 759.

[3] L. Brown et al., Nano Letters, 12 (2012), 1609.

[4] This research was supported by DOE grant DE-FG02-09ER46554 (JL,STP); Wigner Fellowship through the Laboratory Directed Research and Development Program of Oak Ridge National Laboratory, managed by UT-Battelle, LLC, for the U. S. DOE (WZ); Oak Ridge National Laboratory's ShaRE User Facility Program (JCI), which is sponsored by the Office of Basic Energy Sciences, U.S. DOE; the Office of Basic Energy Sciences, Materials Sciences and Engineering Division, U.S. DOE (ARL, SJP, STP); National Science Foundation under award number NSF DMR 0845358 and Army Research Labs (WJF and JK).
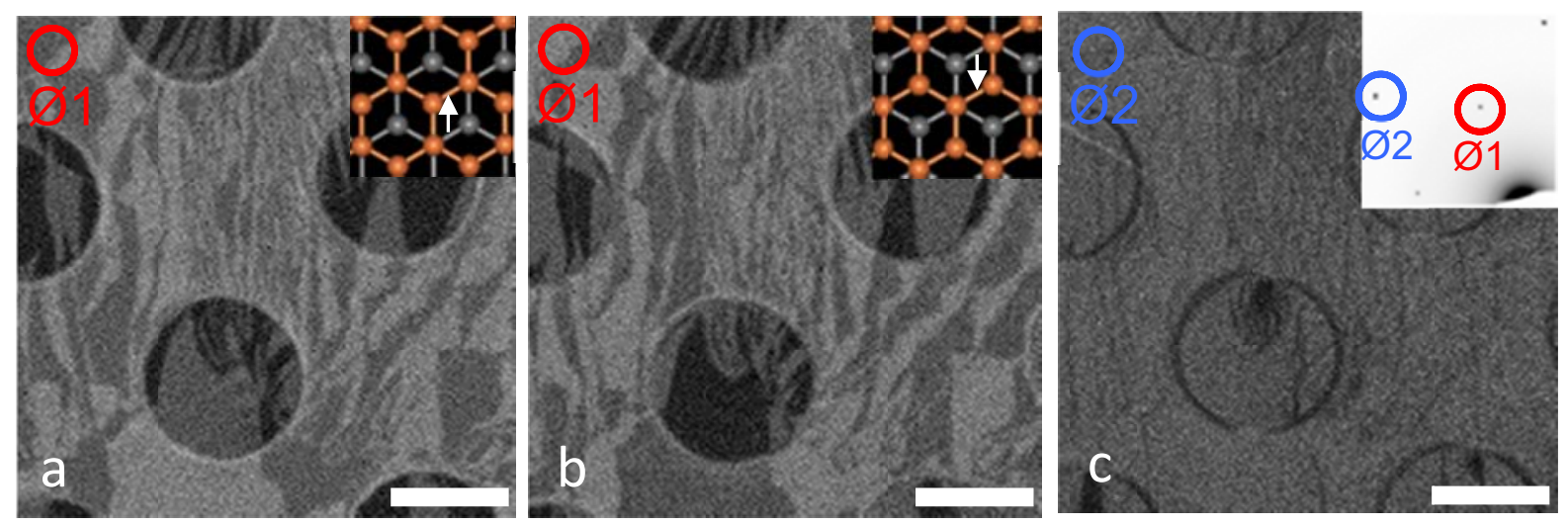

Figure 1. DF-TEM images using the first order diffraction peak Ø1 (red circle) tilted at a) $10^{\circ}$ and b) $10^{\circ}$; and c) the second order diffraction peak $\varnothing 2$ (blue circle) at zero tilt. Inset in a) \& b): Schematic of $\mathrm{AB}$ and $\mathrm{AC}$ stacking in oBLG. Scale bars: $1 \mu \mathrm{m}$.

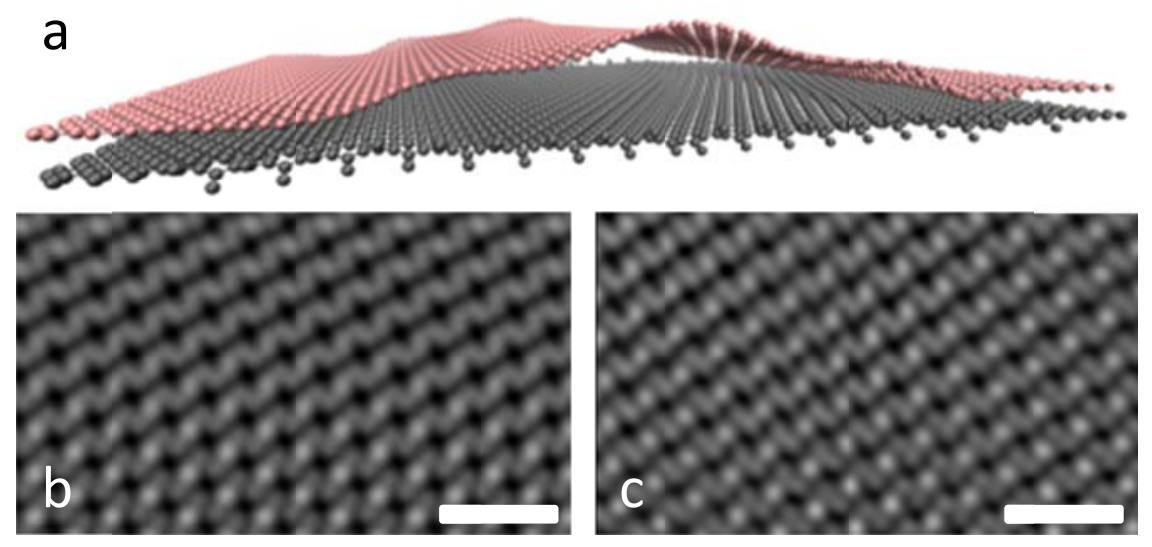

Figure 2. a) Atomic structural model of an $A B / A C$ stacking boundary. b) Simulated STEM image using the above model compared with c) experimental ADF image, both varying gradually across the field of view. Scale bars: $0.5 \mathrm{~nm}$. 